\title{
Dynamics in the Metabasin Space of a Lennard-Jones Glass Former: Connectivity and Transition Rates
}

\author{
Yasheng Yang and Bulbul Chakraborty ${ }^{1}$ \\ ${ }^{1}$ Martin Fisher School of Physics, Brandeis University, \\ Mailstop 057, Waltham, Massachusetts 02454-9110, USA
}

\begin{abstract}
Using simulations, we construct the effective dynamics in metabasin space for a Lennard-Jones glass-former. Metabasins are identified via a scheme that measures transition rates between inherent structures, and generates clusters of inherent structures by drawing in branches that have the largest transition rates. The effective dynamics is shown to be Markovian but differs significantly from the simplest trap models. We specifically show that retaining information about the connectivity in metabasin space is crucial for reproducing the slow dynamics observed in this system.
\end{abstract}

PACS numbers: 64.70.P-, 64.70.kj, 64.70.Q-

\section{INTRODUCTION}

The origin of the dramatic slowing down of dynamics in supercooled liquids upon approaching the glass transition temperature, has been of great research interest. The potential energy landscape (PEL) has proved to be an important conceptual tool for analyzing the dynamics of supercooled liquid [1, 2, 3, 4, 5, 6]. In PEL picture, the configuration hyper space can be seperated into a collection of potential energy valleys, each indentified with a local minimum or "inherent structure". There have been many recent studies demonstrating that the low temperatures dynamics of model supercooled liquids is dominated by activated dynamics between "traps" represented by metabasins which are clusters of inherent structures [7, 8, 9]. In this activated regime, a natural mechanism for glassy dynamics is provided by the trap model[10]. Extensive numerical simulations of model glass formers have shown that the dynamics in metabasin space can be mapped on to the original 11] or an extended version of the trap model[12]. The trap model framework neglects the connectivity in the metabasin space. An interesting question to ask is whether a non-trivial connectivity in the metabasin space, such as the one found in the multiple funnel landscape 13, 14], leads to important differences from the trap model predictions regarding diffusion constants and relaxation timescales.

An important ingredient in any framework for mapping the dynamics of a supercooled liquid to metabasin space is the method for constructing the metabasin. In previous work, metabasins were extracted from molecular dynamics trajectories projected on to the inherent structure space [12], where it was observed that there were long stretches where the system transitioned back and forth between a finite number of inherent structures, and these were grouped into metabasins. The usefulness of the metabasin concept lies in the enormous simplification in the dynamics that results from projecting the supercooled-liquid dynamics on to the metabasin space: this projected dynamics is [12], and therefore, understanding glassy dynamics reduces to the problem of understanding the properties of this random walk. Adopt- ing the perspective that the aim of the metabasin construction is to define a space in which the complex dynamics of the supercooled liquid becomes Markovian, opens up the possibility of constructing metabasins using other algorithms. In this paper, we pursue this line of reasoning and use a metabasin construction scheme based on a knowledge of the transition rates between different inherent structures. With the metabasins defined, we (a) test whether a trap model with these metabasins as the traps provides an adequate description of the observed dynamics of the supercooled liquid, (b) include the connectivity in the metabasin space to extend the trap model predictions and show that the correlations extracted from this random walk, which takes into account the non-trivial connectivity, agrees well with the ones measured directly in the molecular dynamics simulations, and finally (c) we analyze the properties of this random walk in order to gain some insight into the slow dynamics.

\section{MODEL}

The model system we use to study the dynamics of supercooled liquid is a Lennard-Jones binary mixture (LJBM) consisting of 67 particles, 53 of type $\mathrm{A}$ and 14 of type B. The simulation box is a cube with periodical boundary condition. All particles have the same mass $m$, and they interact via a Lennard-Jones potential $v(r)=4 \epsilon\left((\sigma / r)^{12}-(\sigma / r)^{6}\right)$, where $r$ is the distance between them. The interaction parameters, depending on the types of participating particles, are: $\epsilon_{A A}=1.0$, $\sigma_{A A}=1.0, \epsilon_{A B}=1.5, \sigma_{A B}=0.8, \epsilon_{B B}=0.5$ and $\sigma_{B B}=0.88[15]$. In the following, all quantities will be expressed in reduced units, with the unit of length as $\sigma_{A A}$, the unit of energy as $\epsilon_{A A}$, and the unit of time as $\left(m \sigma_{A A}^{2} / \epsilon_{A A}\right)^{1 / 2}[16]$. The number density of particles is 1.2. To accommodate the box with periodic boundary conditions, the potential is shifted and truncated with a quadratic cutoff [16, 17, 18, 19], which ensures continuity of the potential and its gradient at the cutoff radius. 
The modified potential is

$$
\begin{aligned}
v^{c}(r)= & v(r)-v\left(r_{c}\right)-\frac{\left(r^{2}-r_{c}^{2}\right)}{2 r_{c}}\left(\frac{d v(r)}{d r}\right)_{r=r_{c}} \\
= & 4 \epsilon\left((\sigma / r)^{12}-(\sigma / r)^{6}\right. \\
& +\left(6\left(\sigma / r_{c}\right)^{12}-3\left(\sigma / r_{c}\right)^{6}\right)\left(r / r_{c}\right)^{2} \\
& \left.-7\left(\sigma / r_{c}\right)^{12}+4\left(\sigma / r_{c}\right)^{6}\right)
\end{aligned}
$$

for $r<r_{c}$ and $v^{c}(r)=0$ for $r \geq r_{c}$. Since the cutoff distance should be smaller than half of the box size, a cutoff distance $r_{c}=1.9$ is used. This cutoff makes the potential shallower than the original [15], and, therefore, the ratio between $\mathrm{A}$ and $\mathrm{B}$ particles is adjusted to minimize the chances of crystallization. The velocity form of Verlet algorithm is used to solve the Newtonian equation, with $\delta t=0.001$. The temperature range considered is from $T=0.7$ to $T=0.48$. In this regime the supercooled liquid slows down significantly yet its equilibrium properties can be studied in simulations. Temperature is fixed by resampling the velocities from a Boltzmann distribution after every 10240 simulation steps. Initial configurations are generated by first equilibrating the system at $T=5$, then at $T=2$, and then slowly cooling down to the temperatures of interest with cooling rate $\leq 3.33 \times 10^{-6}[20]$ (Smaller cooling rates are used at lower temperatures). At each of these temperatures, the system is equilibrated for a time much longer than (at least an order of magnitude) the estimated $\alpha$-relaxation time before any data is collected for measurement.

\section{INHERENT STRUCTURES}

Inherent structures (IS) are obtained using conjugate gradient minimization techniques during the simulation [3, 6]. Although, theoretically, inherent structures can be labeled by their extact potential energy, there is a danger of labeling two different inherent structures with the same potential energy since any numerical procedures measure the potential energy with finite precision. To alleviate this problem, we use a pair of energies to label each inherent structure, $\left\{V, V_{B B}\right\}$, where $V$ is the potential energy of local minima, and $V_{B B}$ is the potential energy between type $B$ particles. If two different inherent structures have the same $V$, their $V_{B B}$ are likely to be different since they have different arrangement of particles. Implementing this procedure is most important for the metabasin cosntruction since many thousands of IS are generated in the process.

Given an inherent structure, an ensemble of configurations in the same valley can be constructed by using a restricted Boltzmann sampling that is discussed in the next section. With each of these configurations, and initial velocities sampled from the Boltzmann distribution, the waiting time out of this IS valley can be measured using interval-bisection method [9]. For the temperature range considered in this paper, it turns out that for many

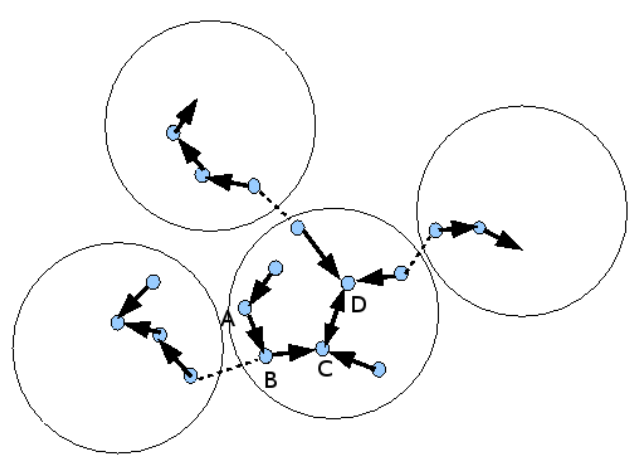

FIG. 1: Illustration of the construction of metabasins. Small circles denote inherent structures. Solid arrows denote the strongest transition branches from corresponding metabasins. Each big circle encloses a cluster of inherent structures, i.e., a metabasin. Dash lines denote the weaker inter-metabasin transitions

inherent structures, the distribution of waiting time deviates from exponential significantly, thus the transition between inherent structures is history-dependent. The history dependence indicates that there is no clear separation of scales between the time taken to equilibrate in an IS valley and the hopping between different IS. This is reasonable given the fact that there can be arbitrarily small dynamical barriers between the IS, which are defined solely based on their property of being a potential energy minimum. It is expected that grouping IS into metabasins such that IS with frequent transitions between them are in the same metabasin would lead to larger barriers between metabasins, and, therefore, a separation of time scales, and Markovian dynamics.

\section{CONSTRUCTION OF METABASINS}

Starting from one inherent structure in a hypothetical metabasin, the system will more likely go into another inherent structure in the same metabasin. To construct a metabasin from a randomly choosen inherent structure $A$, we, therefor, need to start the simulation from $A$ for many times, and count how many times the system goes into each neighboring inherent structure. There must be a most frequently visited neighbor, $B$. Or in another word, $A$ has stronger connection to $B$, than to any of its other neighbors. As shown in Figure 1, $A$ and $B$ get connected by an arrow. The procedure is repeated starting with $B$ and the process of building this cluster continues until we find a connection which links two IS that are already in the cluster. All the IS in the cluster are now assigned to one metabasin. We then check the other neighboring IS found in the process of constructing the cluster and identify their most frequently visited neighbor. If these belong to the existing cluster, then these IS also get assigned to the metabasin containg the starting configuration, $A$. During the construction of metabasin, 
tens of thousands of inherent structures are encountered, which crowd into a small range of potential energy, and as mentioned earlier, it becomes essential to label them with both $\left\{V, V_{B B}\right\}$.

Our construction is similiar to the hierarchical master equation approach in [21]. Comparing with MB construction methods based on trajectories, our approach is objective except for the fact that the computational force is limited to explore all the details of a MB in the phase space.

The construction of the metabasin, described above, relies on the transitions between IS being history independent. It is, therefore, important to obtain an initial ensemble of states which are in equilibrium in the valley $A$ and use these as starting configurations to measure the number of transitions to other IS. All of these starting configurations should have the same set of values for $\left\{V, V_{B B}\right\}$ but a different set of velocities. We implement this restricted Boltzmann sampling by starting from an initial configuration $A_{0}$, with initial velocities sampled from the Boltzmann distribution. and run the simulation for $n$ MD steps. If the final state $A_{1}$ still belongs to the same inherent structure, then we accept it as a member of the ensemble. If not, we count $A_{0}$ again as a member of the ensemble. This process is repeated until we have a large enough ensemble of initial states to measure the transitions from. In practice, $n$ is chosen to be $1 / 4$ of the estimated waiting time of the given IS, and in order to reduce correlation, the above process is repeated 8 times before the resulting state is accepted as initial state. The number of initial states is at least 40 , and more often chosen to be greater for better estimation of branching ratios.

The temperature chosen to construct metabasin is $T=0.52$, a temperature where IS are well defined and the dynamics is not prohibitively slow for metabasin construction. The number of initial inherent structures, randomly chosen from MD trajectories is 140 and each of these constitute the starting point for metabasin construction according to the algorithm described above s.

The potential energy, $E$, of a metabasin is defined to be the energy of the most probable inherent structure in it.

\section{MARKOVIAN PROPERTY}

A hallmark of Markovian processes is that the distribution of waiting times is exponential 22]. Since the motivation behind the construction of the metabasins is obtaining a Markovian model of the dynamics of supercooled liquids, the first task is to check whether our metabasin construction yields a space in which the dynamics is Markovian. To measure the waiting times, an ensemble of initial states is constructed through a restricted Boltzmann sampling in each metabasin and at the temperature of interest. A constant temperature trajectory is then started from each initial state, and interval-bisection

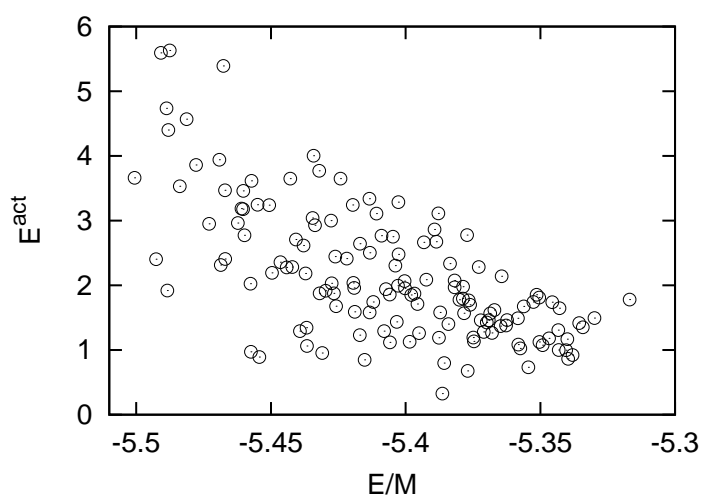

FIG. 2: Effective activation energy $E^{a c t}$ of metabasins, plotted against their energy per particle, $E / M$, where $M$ is the number of particles. The activation energies are calculated according to Eq. (2).

method [9] is used to determine the inherent structure sequence of the trajecotry. If the inherent structures of two succesive quenchs are not in the original metbasin, the system is considered to have made a transition to a new metabasin, and the waiting time is recorded. Since the conjugate gradient method used to minimize the potential energy occasionally leads to a wrong inherent structure, two succesive quenchs are used to signal a transition. For all the metabasins constructed according to our algorithm, the waiting time distributions are founded to be exponential and, therefore, the dynamics in this space is indeed Markovian. The mean waiting time $\tau_{i}$, for a metabasin $i$ with energy $E_{i}$ is measured in the temperature range $0.44 \leq T \leq 0.70$. This mean waiting time is found to be above the ballistic time region, indicating the separation of time scales that we would like to see for metabasins. The mean waiting time is characteristic of an activated process with:

$$
\tau_{i}(T) \approx \tau_{i}^{\infty} \exp \left(E_{i}^{a c t} / T\right)
$$

The effective activation energy $E^{a c t}$ is not strongly correlated with the energy $E$ although $E^{\text {act }}$ tends to be higher for lower lying metabasins, as shown in Figure 2, The time scale, $\tau(T)$ demonstrates a stronger correlation with a marked increase for deeper metabasins, as shown in Figure 3. Since the dynamics in metabasin space is Markovian, the evolution of the probability distribution of metabasins, $P_{i}(t)$, is described by:

$$
\frac{d}{d t} P_{i}(t)=-\frac{1}{\tau_{i}(T)} P_{i}(t)+\sum_{j} w_{i j} P_{j}(t)
$$

where $\tau_{i}$ is the mean waiting time of metabasin $i$ at temperature $T$, and $w_{i j}$ is the transition rate from metabasin $j$ to metabasin $i$. Envisioning the metabasins as traps of depth $E_{i}^{a c t}$, the simplest trap model is one that ignores the connectivity and characterizes the trap space by $\tau_{i}$ 
and a density of states $[10]$ :

$$
\frac{d}{d t} P_{i}(t)=-\frac{1}{\tau_{i}(T)} P_{i}(t)+\omega(t) \rho\left(E_{i}^{a c t}\right)
$$

where $\omega(t)$ is a normalization factor and $\rho\left(E^{a c t}\right)$ is the density of traps with a depth $E^{a c t}$. In most mappings of metabasin dynamics, in Lennard-Jones systems, to trap models the energy of the metabasin, $E$ has been considered as the depth of the trap. As discussed earlier, we find that the activation energy and the nominal energy of a metabasin are not strongly correlated. We, therefore, choose to construct an effective trap model that describes dynamics in an energy landscape, by averaging $\tau_{i}$ over metabasins with energies in a small range around a value $E$ :

$$
\frac{d}{d t} P(E, t)=-\frac{P(E, t)}{\tau(E, T)}+\rho(E, T) \omega(t)
$$

where

$$
\begin{aligned}
P(E, t) & =\sum \delta\left(E_{i}-E\right) P_{i}(t) \\
\frac{1}{\tau(E, T)} & =\frac{\sum_{i} \delta\left(E_{i}-E\right) P_{i, e q}(T) / \tau_{i}(T)}{\sum_{i} \delta\left(E_{i}-E\right) P_{i, e q}(T)} \\
\rho(E, T) & =P_{e q}(E, T) / \tau(E, T) \\
\omega(t) & =\frac{\sum_{i} P_{i}(T) / \tau_{i}}{\sum_{i} P_{i, e q}(T) / \tau_{i}},
\end{aligned}
$$

with $P_{i, e q}(T) \propto \exp \left(-\beta E_{i}\right)$, the equilibrium probability of finding metabasin $i$, and $P_{e q}(E, T)$ the equilibrium distribution of metabasin energy, which can be measured in the simulation. As shown below, this effective trap model differs from the simple trap model [10] in that $\rho(E, T)$ depends on temperature; reflecting the fact that $E_{i}$ is not the trap depth of metabasin $i$.

The metabasin energy distribution $P_{e q}(E, T)$ is obtained from simulations run at $T=0.6,0.52$ and 0.48 , and is found to be well described by a Gaussian over this range [4, 8, 11, 23]. We have extracted the density of states from these distribution and find that $\Omega(E) \propto$ $P_{e q}(E, T) \exp (E / T)$ is, indeed, independent of temperature as show in Figure 5. We use this observation to construct $P_{e q}(E, T)$ at temperatures other than the ones where it was measured explicitly, and combine this information with $\tau(E, T)$ to obtain $\rho(E, T)$. This function, shown in Figure 4 depends on temperature and is different from $\Omega(E)$.

The effective trap model based on the deduced forms of $\rho(E, T), \tau(E, T)$ and $\omega(t))$, provides a description of the activated dynamics near the glass transition. It is, however, a meanfield model that ignores the connectivity in the metabasin space. In order to evaluate the effects of the connectivity on the dynamics, we have calculated various correlation functions using this trap model and compared it to the results of the direct, molecular dynamics simulations.

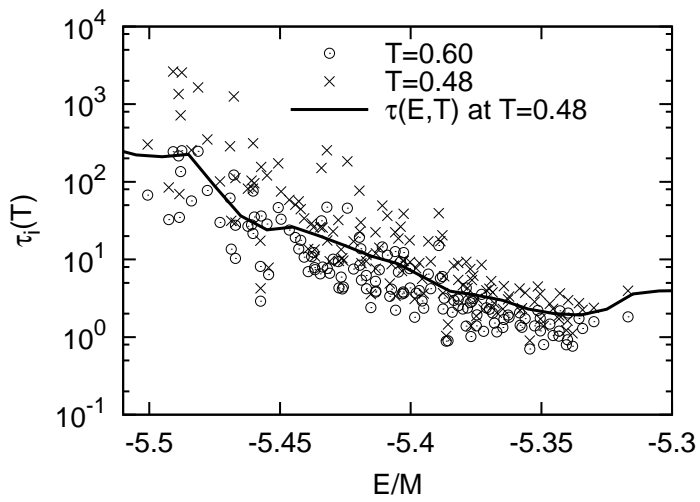

FIG. 3: Waiting time of metabasins measured at $T=0.6$ and 0.48 , plotted against their metabasin energy per particle. The solid line is $\tau(E, T)$ calculated according to Eq. (6)

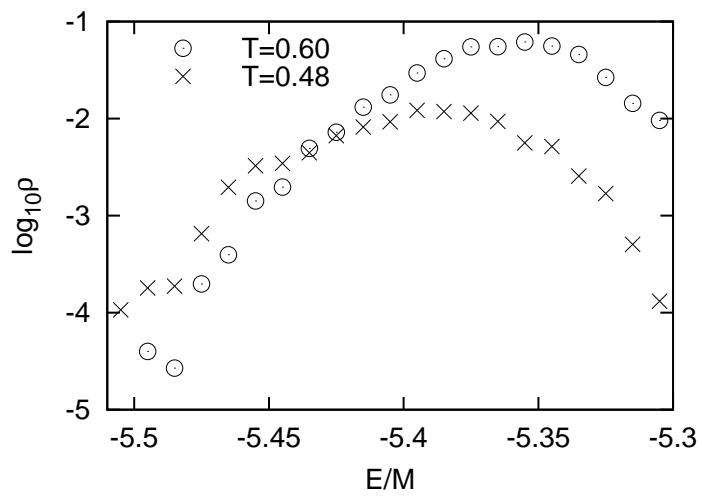

FIG. 4: $\rho(E, T)$ at $T=0.6$ and 0.48 , calculated according to Eq. (6)

\section{TESTING THE TRAP MODEL}

In this section, we compare the predictions of the effective trap model to actual simulation results. In the trap model, correlation functions are calculated by averaging over the sampled metabasins according to:

$$
\begin{aligned}
\langle A\rangle_{e q} & =\sum_{i} P_{i, e q}(T) A_{i} \\
& =\int d E P_{e q}(E, T) \frac{\sum_{i} P_{i, e q}(T) \delta\left(E-E_{i}\right) A_{i}}{\sum_{i} P_{i, e q}(T) \delta\left(E-E_{i}\right)} \\
& =\int d E P_{e q}(E, T) \frac{\sum_{\alpha} \delta\left(E-E_{\alpha}\right) A_{\alpha}}{\sum_{\alpha} \delta\left(E-E_{\alpha}\right)}
\end{aligned}
$$

Here $A$ is the physical observable of interest. The first summation, $\sum_{i}$ is over all metabasins, while $\sum_{\alpha}$ is over the metabasins, sampled according to the Boltzman distribution in the simulations. As mentioned earlier, a total of 140 metabasins were sampled. In numerical calculation, the $\delta$ function in (6), is replaced by a Gaussian with a narrow width, $2 \sigma^{2}=0.01^{2} M^{2}$, with $M=67$ being the number of particles. 


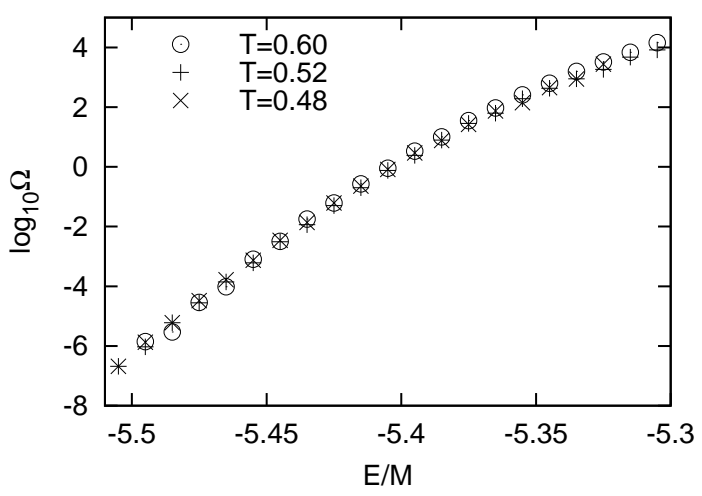

FIG. 5: Plot of the density of states of metabasins, $\Omega(E)$, shifted vertically to make lines overlap.

\section{A. Estimate of the alpha relaxation time}

The $\alpha$ relaxation time $\tau_{\alpha}$ was measured in the simulation through its usual definition: $F_{s}\left(q_{0}, \tau_{\alpha}\right)=1 / e$, where $F_{s}(q, t)$ is the incoherent scattering function 24]. In this paper, $F_{s}(q, t)$ is measured for type $A$ particles and $q_{0}=7.251$ [15]. The $\alpha$ relaxation time can also be estimated from the distribution of single particle displacements [25], which shows marked non-Gaussianity at timescales of the order of $\tau_{\alpha}$. The non-Gaussianity is related to caging and a caging time scale, $\tau_{s}$ can be extracted by measuring the time at which the probability of a type $A$ particle having a translation less than $1 / q_{0}$ diminishes to $1 / e$. As shown in Table \ in the temperature range examined, $\tau_{s}$ and $\tau_{\alpha}$ are proportional to each other. The caged motion of particles has also been related to hopping between metabasins $[9]$.

In the trap model framework, the $\alpha$ relaxation process corresponds to hopping between metabasins [9, 11] , and in this regime, the correlation function $C(t)$ can be constructed by assuming that the correlation is unity when the system is in the same metbasin and drops to zero as it leaves the metabasin[10]. For our effective trap model, this approximation leads to:

$$
C(t)=\left\langle\exp \left(-t / \tau_{i}\right)\right\rangle_{e q}
$$

The expectation value is calculated according to Eq. (7). In Figure 6. $C(t)$ is plotted along with $F_{s}(q, t)$. As expected the shapes of the two functions are different since $C(t)$ has no information about the short time dynamics. For $C(t)$ to be a useful tool for understanding the glass transition, however, the change in time scale of the longtime dynamics should closely resemble that of $\tau_{\alpha}$. Within the trap model, a measure of the $\alpha$ relaxation time is provided by the relaxation time $\tau_{c}$ defined by $C\left(\tau_{c}\right)=1 / e$, and measured using Eq. (8). The values of $\tau_{c}$ along with the values of $\tau_{\alpha}$ are listed in Table $\llbracket$ for $T=0.6,0.52$, and 0.48 and plotted in Figure 7 It is clear that $\tau_{c}$ is much smaller than $\tau_{\alpha}$ for all temperatures listed, and more importantly, $\tau_{\alpha}$ increases significantly faster than $\tau_{c}$. These

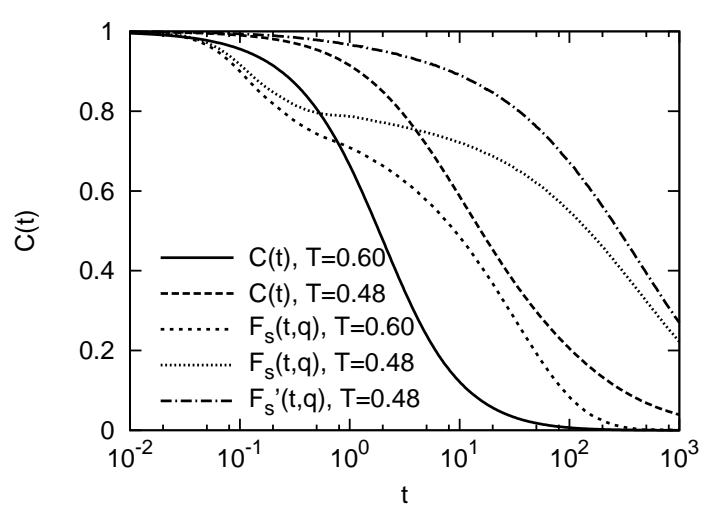

FIG. 6: Comparison of $C(t)$ obtained from the effective trap model at $T=0.6$ and 0.48 and the incoherent scattering function, $F_{s}(q, t)$ measured in simulations. Also shown is $F_{s}^{\prime}(q, t)$, the incoherent scattering function measured from the inherent structure coordinates. Time is measured in scaled units (cf text).

TABLE I: Comparison of estimates of the $\alpha$ relaxation time, $\tau_{\alpha}, \tau_{s}$, and $\tau_{c}$.

\begin{tabular}{c|cccccc}
\hline \hline $\mathrm{T}$ & $\tau_{\alpha}(T)$ & $\tau_{s}(T)$ & $\tau_{c}(T)$ & $\frac{\tau_{\alpha}(T)}{\tau_{\alpha}(0.6)}$ & $\frac{\tau_{s}(T)}{\tau_{s}(0.6)}$ & $\frac{\tau_{c}(T)}{\tau_{c}(0.6)}$ \\
\hline 0.9 & 2.2 & 6.7 & & 0.11 & 0.11 & \\
0.6 & 20 & 62 & 2.9 & 1 & 1 & 1 \\
0.52 & 91 & $2.7 \times 10^{2}$ & 1.1 & 4.5 & 4.4 & 3.8 \\
0.48 & $3.7 \times 10^{2}$ & $1.1 \times 10^{3}$ & 32 & 19 & 18 & 11 \\
0.46 & $8.4 \times 10^{2}$ & $2.5 \times 10^{3}$ & & 42 & 40 & \\
\hline \hline
\end{tabular}

results indicate that the trap model, as defined up to now, does not capture all of the physical processes leading to the slow dynamics.

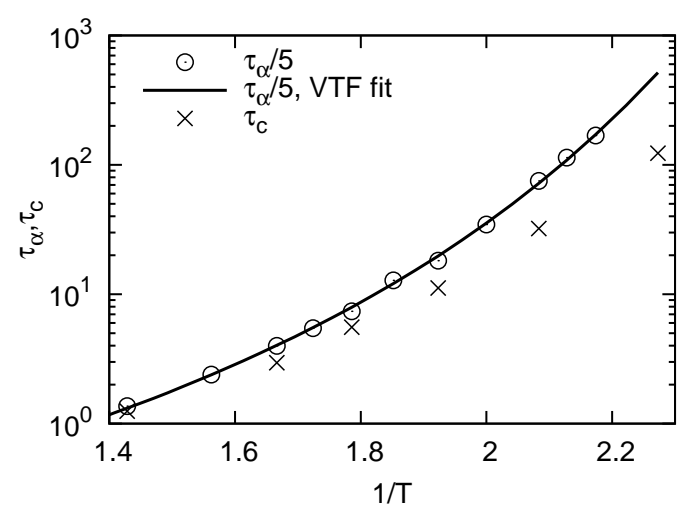

FIG. 7: Temperature dependence of $\tau_{\alpha}$ and $\tau_{c}$, measured in scaled units. The solid line is the fit of $\tau_{\alpha}$ to the VogelTamman-Fulcher form. 


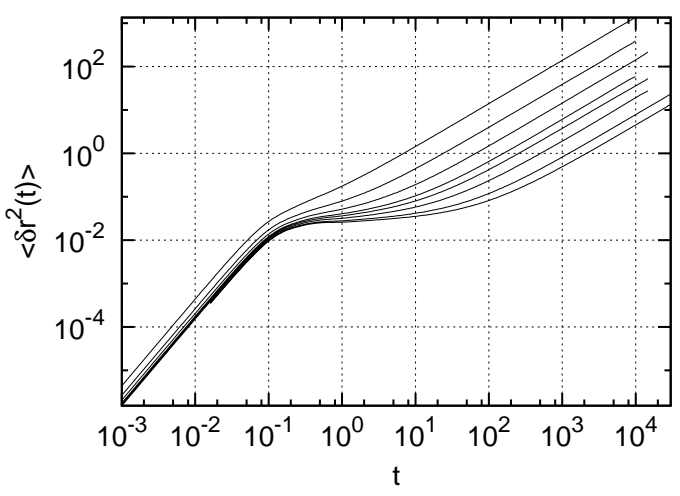

FIG. 8: MSD of type A particles plotted as a function of time $t$ measured in scaled units. The lines correspond to $T=1.5$, $0.9,0.7,0.6,0.56,0.52,0.48$, and 0.46 , top to bottom at $t \geq 1$.

\section{B. Trap model prediction of the mean squared displacement (MSD)}

The MSD $\left\langle\delta r^{2}(t)\right\rangle$ of type A particles is measured directly from the simulations, and as shown in Figure 8 , there is caging. The diffusion constant can be measure by looking at the long-time behavior: $D=\left\langle\delta r^{2}(t)\right\rangle / 6 t$ at large $\left\langle\delta r^{2}(t)\right\rangle$. The temperature dependence of $D$ is shown in Figure 10.

The MSD of particles in space can be mapped on to a random walk between metabasins [9, 26]. Consider a random walk going through a sequence of $N$ metabasins, $\alpha_{1} \rightarrow \alpha_{2} \rightarrow \ldots \rightarrow \alpha_{N+1}$, where $\alpha_{n}$ is the label of metabasin. The metabasin coordinate sequence is labeled as $\vec{\xi}_{1} \rightarrow \vec{\xi}_{2} \rightarrow \ldots \rightarrow \vec{\xi}_{N+1}$. where $\vec{\xi}_{n}$ is a $3 M-$ component vector representing the coordinates of $M$ particles in metabasin $n$. The diffusion constant, $D_{M B}$, of this random walk can be measured from the large $N$ limit:

$$
6 M D_{M B}=\frac{\left\langle\left(\vec{\xi}_{N+1}-\vec{\xi}_{1}\right)^{2}\right\rangle_{r w}}{N\left\langle\tau_{n}\right\rangle_{r w}},
$$

where $\left\langle\tau_{n}\right\rangle_{r w}$ is the average waiting time obtained by averaging over all random walk trajectories in the metabasin space. For each metabasin $\alpha_{n}$, the probability of being at metabasin $i$ is proportional to $P_{i, e q} / \tau_{i}$. Thus $\left\langle\tau_{n}\right\rangle_{r w}$ can be calculated from $\left\langle 1 / \tau_{i}\right\rangle_{e q}$, which is an average over all metabasins weighted with equilibrium probabilities,

$$
\left\langle\tau_{n}\right\rangle_{r w}=\frac{\sum_{i} \frac{P_{i, e q}}{\tau_{i}} \tau_{i}}{\sum_{i} \frac{P_{i, e q}}{\tau_{i}}}=\frac{1}{\left\langle 1 / \tau_{i}\right\rangle_{e q}}
$$

Assuming that at each metabasin transition the displacement in $\vec{\xi}$-space is $\sqrt{\Delta}$, the diffusion constant $D_{M B}$ is, therefore, $D_{M B}=(\Delta / 6 M)\left\langle 1 / \tau_{i}\right\rangle_{e q}$. As shown in Fig. 10. $D_{M B}$ rides above the $D$ measured from the MSD of particles, again, demonstrating that the trap model picture, which ignores all connectivity in the metabasin space, underestimates the slowing down of the dynamics.

\section{EFFECTS OF CONNECTIVITY IN METABASIN SPACE}

One feature of the metabasin space that has been ignored in the trap model is that the Markovian dynamics describes a random walk on a graph with non-trivial connectivity. The calculation of the diffusion constant, $D_{M B}$ can be improved upon by incorporating aspects of the connectivity that are captured by the transition rates $w_{i j}$. Defining, $\delta \vec{\xi}_{n}=\vec{\xi}_{n+1}-\vec{\xi}_{n}$, the mean squared displacement in $\vec{\xi}$-space can be written as:

$$
\begin{aligned}
& \left\langle\left(\vec{\xi}_{N+1}-\vec{\xi}_{1}\right)^{2}\right\rangle_{r w}=\left\langle\left(\sum_{n=1}^{N} \delta \vec{\xi}_{n}\right)^{2}\right\rangle_{r w} \\
& =\sum_{n=1}^{N}\left\langle\left(\delta \vec{\xi}_{n}\right)^{2}\right\rangle_{r w}+2 \sum_{n=2}^{N}\left\langle\delta \vec{\xi}_{n-1} \cdot \delta \vec{\xi}_{n}\right\rangle_{r w} \\
& +2 \sum_{m=2}^{N-1} \sum_{n=m+1}^{N}\left\langle\delta \vec{\xi}_{n-m} \cdot \delta \vec{\xi}_{n}\right\rangle_{r w} \\
& \approx N\left(\Delta+2\left\langle\delta \vec{\xi}_{n-1} \cdot \delta \vec{\xi}_{n}\right\rangle_{r w}\right)
\end{aligned}
$$

Here we ignored $\left\langle\delta \vec{\xi}_{n-m} \cdot \delta \vec{\xi}_{n}\right\rangle_{r w}$ for $m>1$. Then the expression of $D_{M B}$ becomes

$$
6 M D_{M B} \approx\left\langle 1 / \tau_{i}\right\rangle_{e q}\left(\Delta+2\left\langle\delta \vec{\xi}_{n-1} \cdot \delta \vec{\xi}_{n}\right\rangle_{r w}\right)
$$

To calculate $\left\langle\delta \vec{\xi}_{n-1} \cdot \delta \vec{\xi}_{n}\right\rangle_{r w}$, one first picks the metabasin $\alpha_{n}$ at time step $n$ to be a specific one, say $i$, and averages over all possible metabasins $j$ at step $n-1$, and over all possible metabasins $j^{\prime}$ at step $n+1$, followed by averaging over all possible metabasins $i$ at step $n$. For a particular $\alpha_{n}=i$, the probability of having a metabasin $j^{\prime}$ at $n+1$ is $w_{j^{\prime} i} / \sum_{k} w_{k i}=w_{j^{\prime} i} \tau_{i}$. Similarly, the probability of having a metabasin $j$ at step $n-1$ must be $w_{j i} \tau_{i}$. Thus

$$
\begin{aligned}
\left\langle\delta \vec{\xi}_{n-1} \cdot \delta \vec{\xi}_{n}\right\rangle_{i} & =\left\langle\delta \vec{\xi}_{n-1}\right\rangle_{i} \cdot\left\langle\delta \vec{\xi}_{n}\right\rangle_{i} \\
& =\left(\sum_{j} w_{j i} \tau_{i} \delta \vec{\xi}_{i j}\right) \cdot\left(\sum_{j^{\prime}} w_{j^{\prime} i} \tau_{i} \delta \vec{\xi}_{j^{\prime} i}\right)
\end{aligned}
$$

with $\delta \vec{\xi}_{i j} \equiv \vec{\xi}_{i}-\vec{\xi}_{j}$. Two facts should be noticed, (1) the dimension of $\vec{\xi}$, i.e., the number of independent coordinates, $d=3 M-3=198$, is very high, and (2) For many metabasins the connectivity is sparse, with strong connections only to one other metabasin. An example is provided by the metabasins A and B in Table I. Because of (1), any pair $\delta \vec{\xi}_{i j}$ and $\delta \vec{\xi}_{j^{\prime} i}$ with $j^{\prime} \neq j$ can be considered as two random vectors in a high dimensional space, and hence, $\delta \vec{\xi}_{i j} \cdot \delta \vec{\xi}_{j^{\prime} i} \approx 0$. The exception is of course when $j=j^{\prime}$, i.e., when the system hops back and forth between two metabasins $i$ and $j$, and $\delta \vec{\xi}_{i j} \cdot \delta \vec{\xi}_{j^{\prime} i}=-\left(\delta \vec{\xi}_{j i}\right)^{2}=-\Delta$. So we have

$$
\begin{aligned}
\left\langle\delta \vec{\xi}_{n-1} \cdot \delta \vec{\xi}_{n}\right\rangle_{i} & \approx-\sum_{j}\left(w_{j i} \tau_{i}\right)^{2} \Delta \\
& =-p_{i}^{2} \Delta
\end{aligned}
$$


TABLE II: The branching ratio, $w_{j i} \tau_{i}$ of four different metabasins at $T=0.52$

\begin{tabular}{c|cccc}
\hline \hline $\mathrm{i}$ & $\mathrm{A}$ & $\mathrm{B}$ & $\mathrm{C}$ & $\mathrm{D}$ \\
\hline$w_{j i} \tau_{i}$ & 0.87 & 0.61 & 0.11 & 0.13 \\
& 0.05 & 0.24 & 0.06 & 0.07 \\
& 0.02 & 0.06 & 0.05 & 0.05 \\
& 0.007 & 0.01 & 0.04 & 0.04 \\
& 0.006 & 0.01 & 0.04 & 0.03 \\
& 0.003 & 0.01 & 0.04 & 0.03 \\
& $\ldots$ & $\ldots$ & $\ldots$ & $\ldots$ \\
\hline$p_{i}^{2}$ & 0.76 & 0.43 & 0.03 & 0.04 \\
\hline \hline
\end{tabular}

where $p_{i}^{2} \equiv \sum_{j}\left(w_{j i} \tau_{i}\right)^{2}$ is the probability of return to the previous metabasin. For those metabasins that have many neighbors, each with similiar (and low) branching ratio $w_{j^{\prime} i} \tau_{i}$, such as metabasin $\mathrm{C}$ and $\mathrm{D}$ in Table $\mathrm{II}$, the return probability is small.

Averaging Eq. (14) over all possible metabasins $i$, one obtains,

$$
\begin{aligned}
\left\langle\delta \vec{\xi}_{n-1} \cdot \delta \vec{\xi}_{n}\right\rangle_{r w} & =\frac{\sum_{i} \frac{P_{i, e q}}{\tau_{i}}\left\langle\delta \vec{\xi}_{n-1} \cdot \delta \vec{\xi}_{n}\right\rangle_{i}}{\sum_{i} \frac{P_{i, e q}}{\tau_{i}}} \\
& \approx-\frac{\Delta\left\langle p_{i}^{2} / \tau_{i}\right\rangle_{e q}}{\left\langle 1 / \tau_{i}\right\rangle_{e q}}
\end{aligned}
$$

and the expression for the diffusion constant becomes

$$
D_{M B} \approx \frac{\Delta}{6 M}\left\langle\left(1-2 p_{i}^{2}\right) / \tau_{i}\right\rangle_{e q}
$$

The branching ratios $w_{j i} \tau_{i}$ are measured for all the sampled metabasins, and $p_{i}^{2}$ s are calculated. As shown by the examples in Figure 9, the return probability $p_{i}^{2}$ is relatively low at high temperatures. But, as the temperature decreases, $p_{i}^{2}$ increases significantly for some metabasins. This is because some of the transition branches are suppressed as temperature decreases. According to Eq. (16), high $p_{i}^{2}$ makes the diffusion constant smaller. Increasing values of $p_{i}^{2}$ is, therefore, another source of the slow dynamics, and one that is not captured in the trap model.

In Figure 10, the prediction of Eq. (16) is compared to measured $D(T)$. It shows that incorporating the return probability $p_{i}^{2}$ has a significant effect on $D_{M B}$ and improves the agreement with the measured $D$. In [9], Doliwa and Heuer were able to predict the diffusion constant without considering the return probability, due to the fact that the metabasins were constructed from trajectories, and the inherent structures that were connected by quick hops were grouped together into the same metabasin. The dynamics in the space of MB defined in this manner satisfies more stringent requirements [27], inducing $p_{i}^{2}=0$. In this work, metabasins were instead constructed from the transition branch ratio of inherent structures, without any consideration of the absolute values of the barrier heights between metabasins. Our

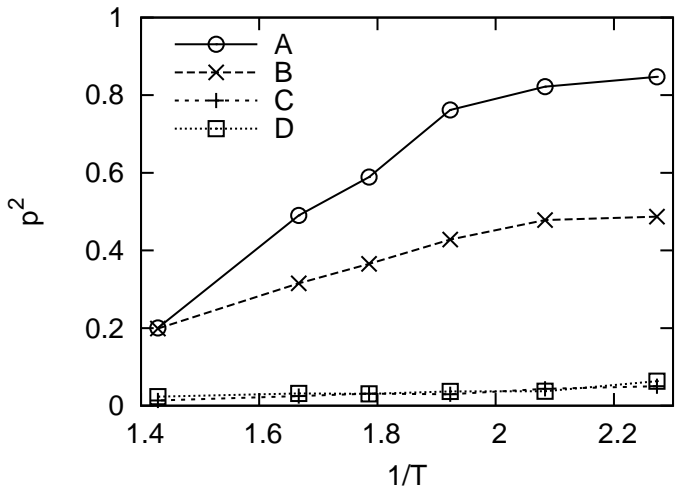

FIG. 9: Comparison of $p_{i}^{2}(T)$, the return probabilities of the four metabasins listed in Table II]

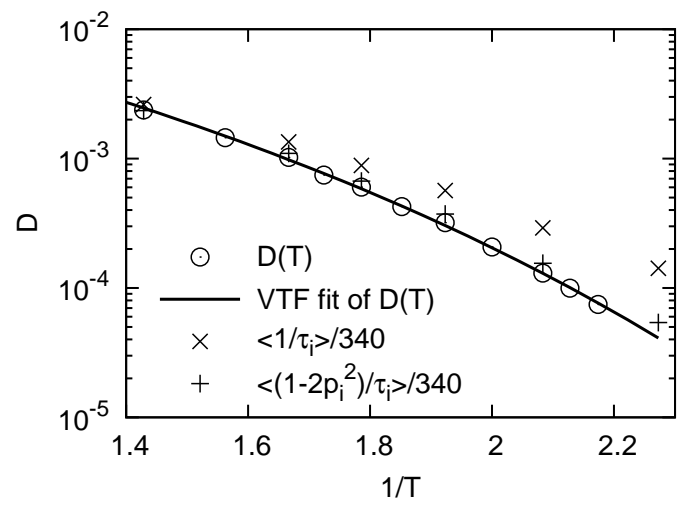

FIG. 10: Comparison of the temperature dependence of the measured diffusion constant, $D$, of type $A$ particles, with the predictions of the trap model, and the trap model modified to include a finite return probability. The relationship between the diffusion constant and $\tau_{i}$ in Eq. 16 has an undetermined constant, which was chosen to be $1 / 340$ in this figure.

construction gives rise to a graph which is much more inhomogeneous and the mean-field assumption of the trap model is not a good approximation. The advantage of this method of construction is that it gives a better idea of the connectivity. To emphasize, the effective dynamics in the metabasin space is certainly Markovian but the random walk lives on a graph with non-trivial connectivity.

\section{SUMMARY}

In this work, we have presented a technique for constructing metabasins using branching ratios of transitions between inherent structures. The dynamics in the space of these metabasins is Markovian, and can be described as an activated process in an energy landscape. A mean field trap model that ignores the connectivity of the metabasins, however, underestimates the slowing down of the dynamics with decreasing temperature. We 
show that incorporating the connectivity through a set of return probabilities leads to quantitative agreement between the predicted and measured temperature dependence of the diffusion constant. This result demonstrates that connectivity is an essential element of the effective dynamics in the metabasin space. At temperatures higher than $\simeq 0.7$, the mean waiting time of metabasins approachs the ballistic time scale, and mapping the dynamics to metabasin space loses its utility. At temperatures lower than $\simeq 0.45$, the return probabilities become so large that higher order terms in Eq.(11) need to be included and the effective dynamics loses its simplicity. The mapping to metabasin space is, therefore, most useful in the range $0.45<T<0.7$ At the high end of this temperature range, the connectivity is reasonably homogeneous, and the return probabilities are low. In this regime, effective trap models capture the essential features of the slow dynamics. As the system is cooled, some of the connections are suppressed more strongly then the others. The connectivity becomes sparse, resulting in higher return probabilities, and break down of the assumption, underlying the trap model, that once the system escapes fro a trap it chooses one from a fixed distribution of trap depths that does not have any connectivity information. It is clear that the difference between con- nections, i.e., the inhomogeniety of connectivity, causes the connectivity to be sparse at low temperatures. At the same time, we find no obvious correlation between return probability and metabasin energy, and therefore, metabasins of different energies seems to have similiar connectivity environment.

Instead of metabasins, J. Kim and T. Keyes 28] have considered the strong return dynamics between inherent structures of a supercooled $\mathrm{CS}_{2}$ system, and postulated that the return dynamics could be coarse-grained to the motion within metabasins. In this paper, however, we showed that even after coarse-graining the strongly connected inherent structures into metabasins at a temperature in the middle of the range of interest to the slow dynamics, the connectivity between metabasins plays a significant rule, especially at low temperatures. Return dynamics appears to be less important for some small systems 29]. In general, however, if the multiple funnel landscape [13, 14] is an adequate representation of the energy minima, then any definition of metabasins based on a single temperature will have to include the influence of return dynamics at low enough temperatures.

We acknowledge helpful discussions with David Reichman, especially during the early stages of this work. This work was supported by NSF DMR-0549762.
[1] M. Goldstein, J. Chem. Phys. 51, 3728 (1969).

[2] F. H. Stillinger and T. A. Weber, Science 225, 983 (1984).

[3] F. H. Stillinger, Science 267, 1935 (1995).

[4] S. Büchner and A. Heuer, Phys. Rev. E 60, 6507 (1999).

[5] T. B. Schrøder, S. Sastry, J. C. Dyre, and S. C. Glotzer, J. Chem. Phys. 112, 9834 (2000), cond-mat/9901271.

[6] A. Heuer, Journal of Physics: Condensed Matter 20, 373101 (2008).

[7] S. Büchner and A. Heuer, Phys. Rev. Lett. 84, 2168 (2000).

[8] B. Doliwa and A. Heuer, Phys. Rev. Lett. 91, 235501 (2003).

[9] B. Doliwa and A. Heuer, Phys. Rev. E 67, 030501 (2003), cond-mat/0205283.

[10] C. Monthus and J.-P. Bouchaud, Journal of Physics A Mathematical General 29, 3847 (1996), condmat/9601012.

[11] R. A. Denny, D. R. Reichman, and J.-P. Bouchaud, Phys. Rev. Lett. 90, 025503 (2003).

[12] A. Heuer, B. Doliwa, and A. Saksaengwijit, Phys. Rev. E 72, 021503 (2005), cond-mat/0503211.

[13] J. Doye, M. Miller, and D. Wales, J. Chem. Phys. 111, 8417 (1999).

[14] D. Wales, J. Doye, M. Miller, P. Mortenson, and T. Walsh, Advances in Chemical Physics 115, 1 (2000).

[15] W. Kob and H. C. Andersen, Phys. Rev. Lett. 73, 1376 (1994).

[16] M. P. Allen and D. J. Tildesley, Computer simulation of liquids (Clarendon Press, New York, NY, USA, 1987),
ISBN 0-19-855375-7.

[17] S. D. Stoddard and J. Ford, Phys. Rev. A 8, 1504 (1973).

[18] A. S. S and S. Sastry, Journal of Physics Condensed Matter 15, 1253 (2003).

[19] D. Coslovich and G. Pastore, J. Chem. Phys. 127, 124504 (2007), 0705.0626.

[20] S. Sastry, P. G. Debenedetti, and F. H. Stillinger, Nature (London) 393, 554 (1998).

[21] J. C. Mauro, P. K. Gupta, and R. J. Loucks, The Journal of Chemical Physics 126, 184511 (pages 11) (2007), URL http://link.aip.org/link/?JCP/126/184511/1.

[22] N. G. V. Kampen, Stochastic processes in physics and chemistry (North-Holland, Amsterdam, 1992).

[23] F. Sciortino, W. Kob, and P. Tartaglia, Phys. Rev. Lett. 83, 3214 (1999).

[24] J. P. Hansen and I. R. McDonald, Theory of Simple Liquids (Academic Press, London, 1986).

[25] G. Szamel and E. Flenner, Phys. Rev. E 73, 011504 (2006), arXiv:cond-mat/0508108.

[26] B. M. Schulz, S. Trimper, and M. Schulz, J. Chem. Phys. 114, 10402 (2001).

[27] O. Rubner and A. Heuer, Physical Review E (Statistical, Nonlinear, and Soft Matter Physics) 78, 011504 (pages 4) (2008), URL http://link.aps.org/abstract/PRE/v78/e011504

[28] J. Kim and T. Keyes, The Journal of Chemical Physics pp. 4237-4245 (2004).

[29] T. Keyes and J. Chowdhary, Phys. Rev. E 64, 032201 (2001). 